\title{
OPERATION OF THE APS PHOTOINJECTOR DRIVE LASER SYSTEM
}

\author{
Yuelin Li
}

December 2004

\begin{abstract}
The APS photoinjector drive laser system has been in operation since 1999 and is achieving a performance level exceeding the requirement of stable operation of the LEUTL FEL system. One remarkable number is the UV energy stability of better than $2 \% \mathrm{rms}$, sometimes less than $1 \%$ rms. This report summarizes the operation experience of the laser system and the improvements made along the way. We also outline the route of upgrade of the system and some frontier laser research and development opportunities in ultrabright electron beam generation.
\end{abstract}

\section{Introduction}

A photoinjector is the only way to generate the high-brightness beams required by fourthgeneration light sources. However, most of the existing photoinjector drive laser systems, the starting point of everything, are obsolete flash-lamp-pumped systems [1], including the GTF drive laser at SLAC and the ATF drive laser at BNL. Funding constraints limit the near-term possibility of replacing these lasers with diode-pumped drivers, hence it is important to understand the common pitfalls and maintain these systems so they can efficiently support the research on high-brightness beams and future light sources.

The APS photoinjector drive laser (APSPDL), in particular, is a glass laser that has significant thermal lensing effects associated with the poor thermal conductivity of the glass, which limit the achievable performance. Despite all the problems we encountered in the past few years, the APSPDL has been a workhorse for the operation of the LEUTL FEL system, leading to the demonstration of optical SASE FEL [2], demonstration of saturation operation of a SASE FEL [3], thorough characterization of the SASE FEL pulse [4-7] and some exciting applications [8]. The system is operating with a performance exceeding the requirement of the FEL operation.

\section{The APS Laser System}

\subsection{The laser system}

The APS laser system, consisting of a Time-Bandwidth Product GLX-200 oscillator and a Positive Light flash-lamp-pumped Nd:Glass amplifier, is a generic chirped-pulse amplification (CPA) system. In a CPA system, the short pulse from the oscillator is first stretched and then amplified and recompressed. The recompressed infrared (IR) pulse at $1053 \mathrm{~nm}$ is subsequently frequency-quadrupled to obtain the ultraviolet (UV) pulse at $263 \mathrm{~nm}$. The UV pulse is relayed to the cathode of the photoinjector to generate the electron beam.

A block diagram of the APSPDL is shown in Fig. 1. The basic operational parameters of the system are listed in Table 1. 


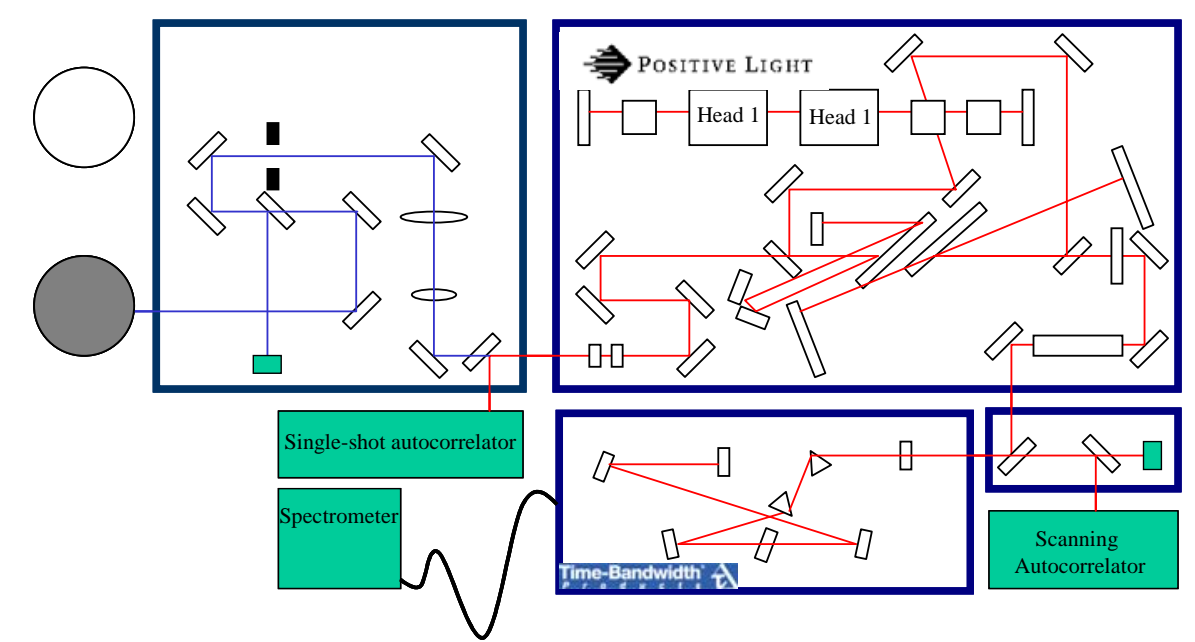

Fig. 1: A block diagram of the APS photoinjector drive laser system.

Table 1: Basic Parameters of the APS Photoinjector Drive Laser System

\begin{tabular}{|l|l|l|l|l|}
\hline & Repetition rate & \multicolumn{1}{|c|}{ Energy/power } & Pulse length & Timing jitter \\
\hline $\begin{array}{l}\text { Oscillator } \\
\text { (tbwp GLX 200) }\end{array}$ & $119 \mathrm{MHz}$ & $120 \mathrm{~mW} @ 1053 \mathrm{~nm}$ & $200 \mathrm{fs}$ & $200 \mathrm{fs}$ \\
\hline Amplifier & $6 \mathrm{~Hz}$ & $\begin{array}{l}6 \mathrm{~mJ} @ 1053 \mathrm{~nm} \\
0.4 \mathrm{~mJ} @ 263 \mathrm{~nm}\end{array}$ & $2-10 \mathrm{ps}$ & \\
\hline
\end{tabular}

\subsection{The oscillator and timing stability}

The oscillator is based on the SESAM technique [8] and is time stabilized to the reference clock of $119 \mathrm{MHz}$ (24th subharmonic of the S-band rf of the linac at $2856 \mathrm{MHz}$ ) through a piezo/pico motor assembly.

The specified timing jitter is 2 ps. A few attempts were made to measure the timing jitter. The most recent measurement was carried out in collaboration with MIT, revealing an rms jitter of 0.5-1 ps (Fig. 2) with respect to the linac rf. The oscillator is pumped by two diode bars, and has been very stable and reliable since its installation, hence has not been the main focus of the maintenance/improvement effort.

The available diagnostics for the oscillator include a power meter monitoring the energy stability, a CCD camera for the beam profile, a scanning autocorrelator (Positive Light Femtoscope) for the pulse duration, and a spectrometer to monitor the spectrum.

\subsection{The amplifier cavity}

The pulses from the oscillator are stretched and injected into the regenerative amplifier cavity, the most troublesome part of the whole laser system. 


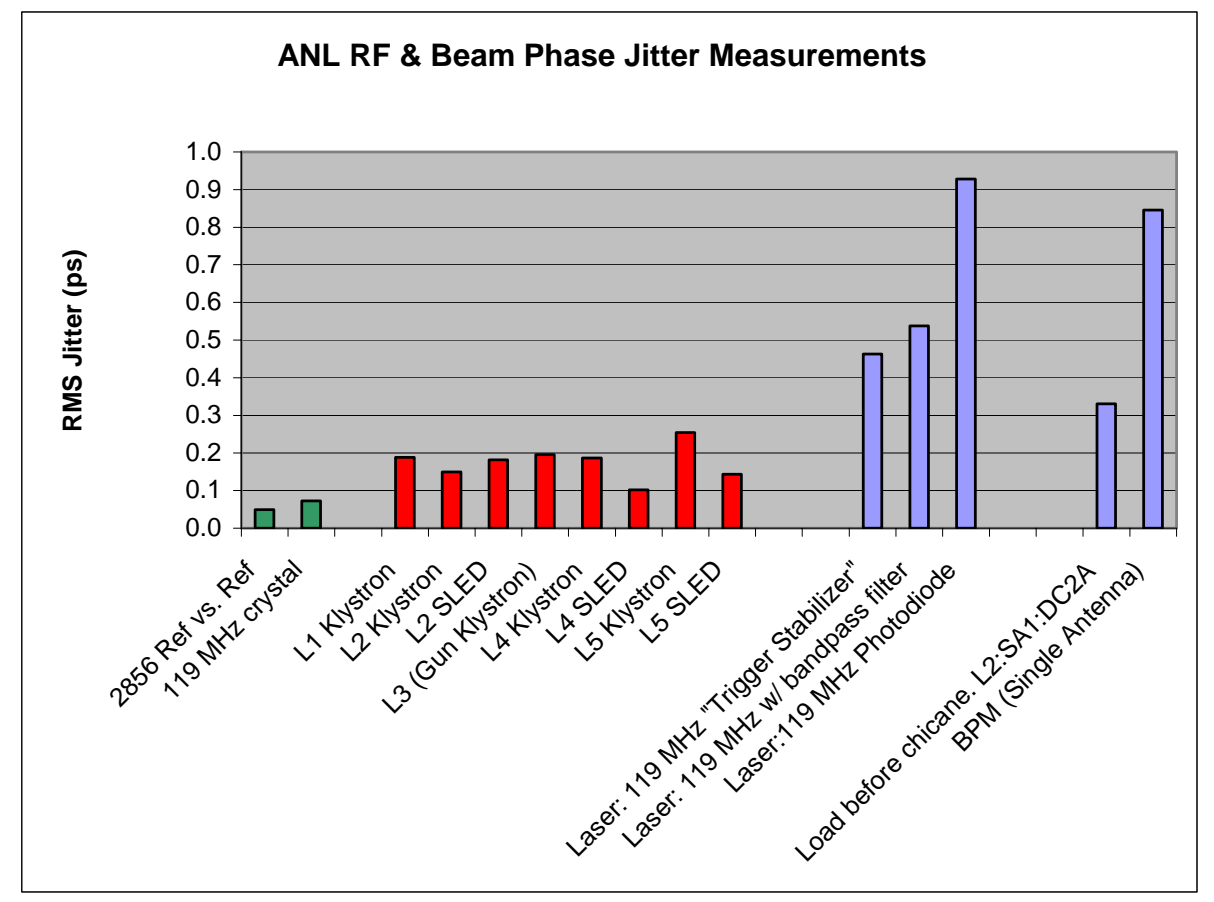

Fig. 2: Timing jitter measurement for the APS injector linacs and the photinjector drive lasers. The result for the laser ranges from 0.5 to $1 \mathrm{ps}$ rms depending on where the laser signal is taken. Collaboration with MIT.

The cavity is a generic regenerative cavity consists of two flash-lamp-pumped Nd: Glass heads, which are responsible for most of the problems we encounter. The laser rods in the heads are 6 $\mathrm{cm}$ long and $4.5 \mathrm{~mm}$ in diameter. Because of these glass heads, the APSPDL is one of the only two such systems in the world. The other one is the GTF PDL at SLAC with a slightly different configuration and a lower repetition rate of $2 \mathrm{~Hz}$.

The first problem we had was optical damage of the laser rods and the Pockel cells. There were two types of optical damage. One was surface damage and the other was internal damage. We were not able to accurately locate the source of either type of damage, but in general we understand they were due to faulty cavity configuration and minor misalignment of the cavity. The deeper root causes may be due to the thermal lensing effect of the long glass rods. This effect can vary for different pump energies and for different melts of the glass.

To mitigate/avoid the problem, the cavity was reconfigured a few times, and the heads were redesigned to increase the pumping efficiency and reduce the thermal load. An alignment procedure has been carefully followed to minimize the cavity misalignment that was found mostly associated with surface damage of the laser rods.

The second problem was chemical and mechanical aging of the laser rods, which eventually lead to poor laser performance and breakage of the rods. The degradation of the laser performance due to aging is most clearly shown in Fig. 3, which gives the energy as a function of time at 1 million and 10 million shots on the laser rods. The degradation is speculated to be due to 

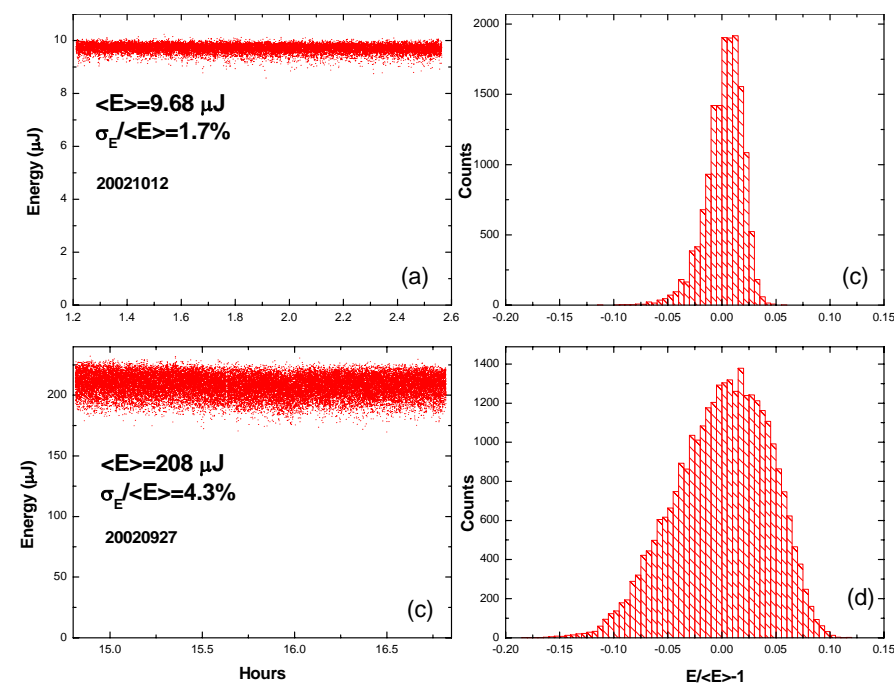

Fig. 3: Measurement of the energy output at $263 \mathrm{~nm}$ as a function of time at (a) 1 million shots of use and (c) 10 million shots of use of a pair of laser rods. The corresponding histograms are also given. Note that the different energy levels were obtained by optical attenuation.

solarization by the flash lamp radiation and doping depletion by the coolant deionized water, etc., but we cannot confirm this so far.

The aging of the flash lamps can also contribute to this behavior, though less likely with our operational parameters. With our operation, the vendor expert expects that the lamps should last 10 billion shots. We did confirm the effect of the flash lamp aging, which normally can be compensated by increasing the pump energy on the lamps. In our case this was difficult to do due to the changing thermal load on the laser rods, which in turn lead to significant changes in laser beam profile and divergence.

After 10 millions shots, the rods broke at the gas kits that seal the cooling water. This mechanical breakage was apparently the result of the mechanical stress build-up over time at the cold/hot interface due to the thermal load. So far we have tried two vendors for the laser rods, Schott (Part \# APG1 LB1061 LK 3545 D9777 5\%, 2 deg/2deg parallel) and Kigre (Q-98 6\%) products. They both show the same behavior.

To get around this problem, we established a maintenance schedule in replacing the laser rods and the flash lamps. A cavity alignment procedure is carefully followed each time the rods and the flash lamps are replaced. By following the alignment procedure, minimum misalignment is achieved.

\subsection{The compressor}

The 300-ps pulse from the amplifier was collimated and sent into the compressor to generate 1to 10 -ps pulses. The original stretcher/compressor gratings were $1800 \mathrm{~g} / \mathrm{mm}$ and had very low 
throughput of about 33\%. This significantly impacted the performance of the laser system with respect to stability.

We replaced the gratings with $1760 \mathrm{~g} / \mathrm{mm}$ gratings from Jobin Yvon with one-pass efficiency of $>91 \%$, and thus about $68 \%$ of total throughput efficiency. This greatly helped us in stabilizing the UV energy output, as will be seen later.

At this stage, we have a CCD camera and a single-shot autocorrelator to monitor the pulse length and the beam profile. We have also taken frequency-resolved optical gating (FROG) measurement of the IR pulse at the output of the compressor (Fig. 4).

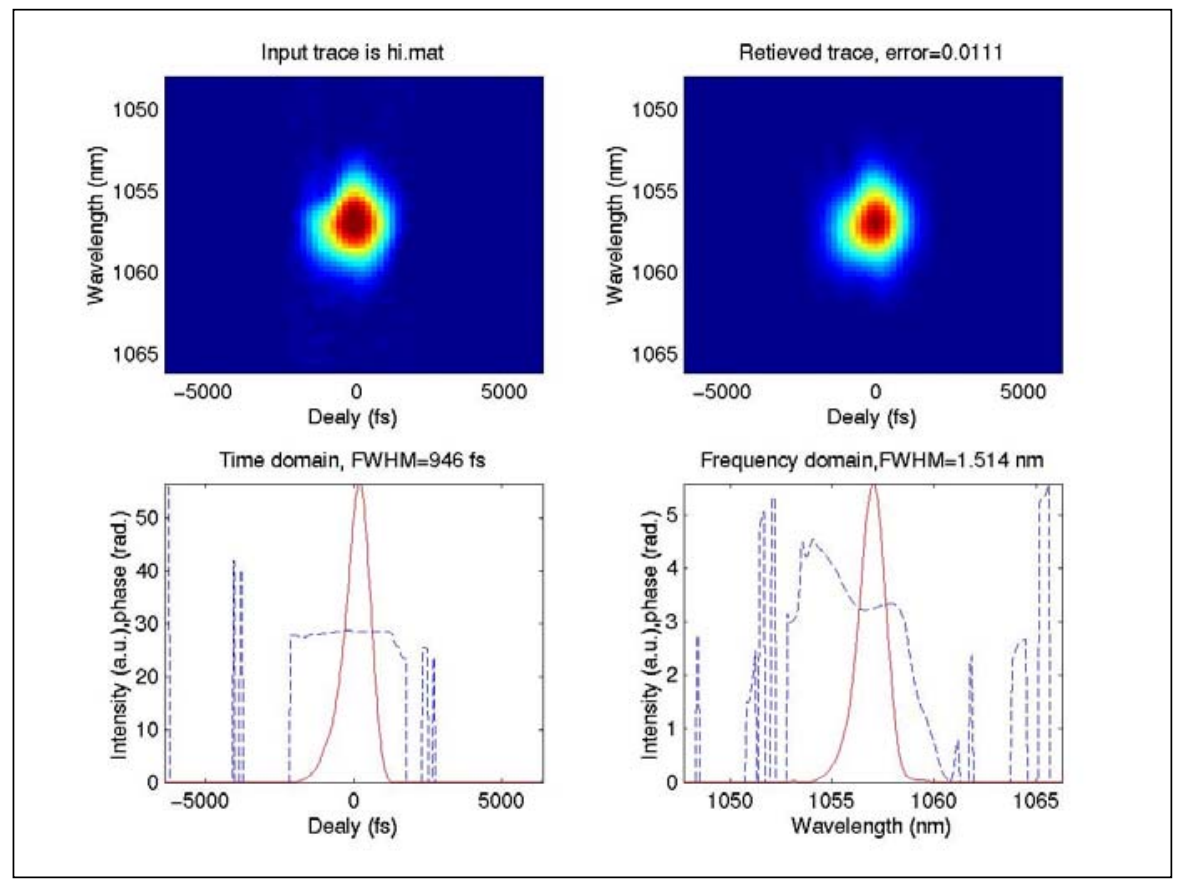

Fig. 4: FROG measurement of the IR output pulse at $1053 \mathrm{~nm}$ from the amplifier after compression.

\subsection{Frequency conversion}

The laser was originally equipped with KDP crystals using noncritical phase matching. Because of the large thickness of the crystal $(2 \mathrm{~cm})$, the beam profile was significantly degraded. Furthermore, the conversion efficiency was only $7 \%$.

To improve the beam quality and the conversion efficiency, we replaced the crystals with two 1mm BBO crystals. To further improve the stability, the beam was down-collimated to about 1$\mathrm{mm}$ for higher intensity at the crystals. Those measures, together with the higher efficiency in the compressor, helped us push the conversion to the linear regime and substantially stabilized the output at $263 \mathrm{~nm}$. The energy jitter is routinely smaller than $2 \%$ rms, sometimes smaller than $1 \%$. Figure 5 illustrates the theoretical conversion efficiencies as a function of the 1053-nm input energy for our system. The overall conversion efficiency we obtained is $15 \%$. 


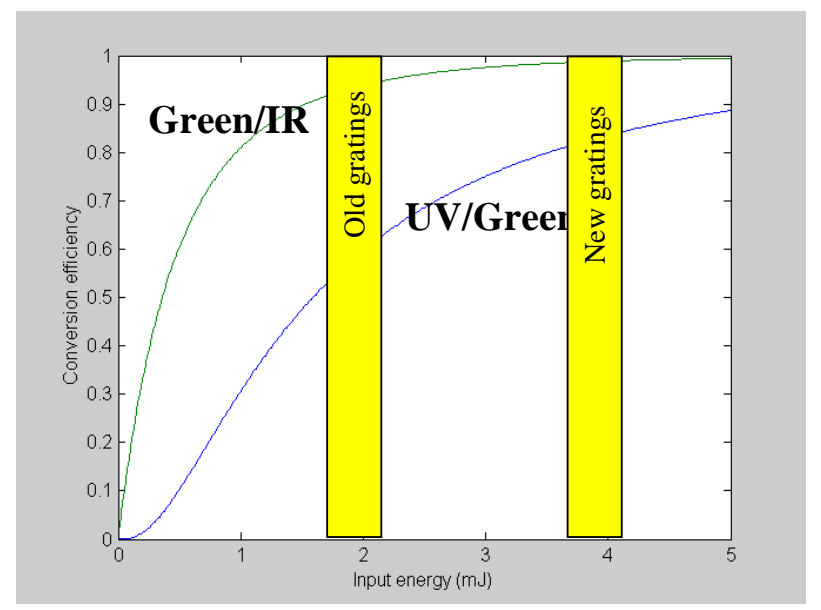

Fig. 5: The theoretical conversion efficiencies of IR to green and green to UV as a function of the input IR pulse energy. The shed area shows the regime with old and new grating set for the compressor in the laser system.

We observed interdependence between the crystal incidence angle, the beam profile, and the compress grating setting. For example, the UV pulse energy after apertures with different sizes can be optimized at different settings of the crystal angle (especially the green/UV one) and the compressor. We believe this has to do with the optics in the laser and the compressor and originated from the limited acceptance angle of the BBO crystals.

\subsection{UV transport}

The inherent laser pointing stability and beam divergence are on the order of $1 \mathrm{mrad}$, as specified by the vendor. This clearly poses the need for image relay to maintain the pointing stability and the beam size.

We implemented a one-lens image relay system to relay the image of an aperture directly onto the photocathode. In doing so, the pulse forms a waist somewhere down the transport line and the local high intensity induces a nonlinear effect in the air, which destroys the fidelity of the laser pulse both longitudinally and transversely.

To mitigate this problem, we filled the transport line with He gas. Helium was chosen because it has the highest ionization potential and smallest nonlinear refractive index. This solved the problem.

As a final diagnostic, a beam splitter takes part of the laser beam onto a CCD camera at a plane equivalent to the cathode of the injector. We call the camera "virtual cathode."

The beam profiles taken for the IR at the compressor exit and the virtual cathode are given in Fig. 6. We have very nice Gaussian shape both in IR and in the UV, though the clipped Gaussian is clearly far from the flat-top profile desired for small emittance beams. 

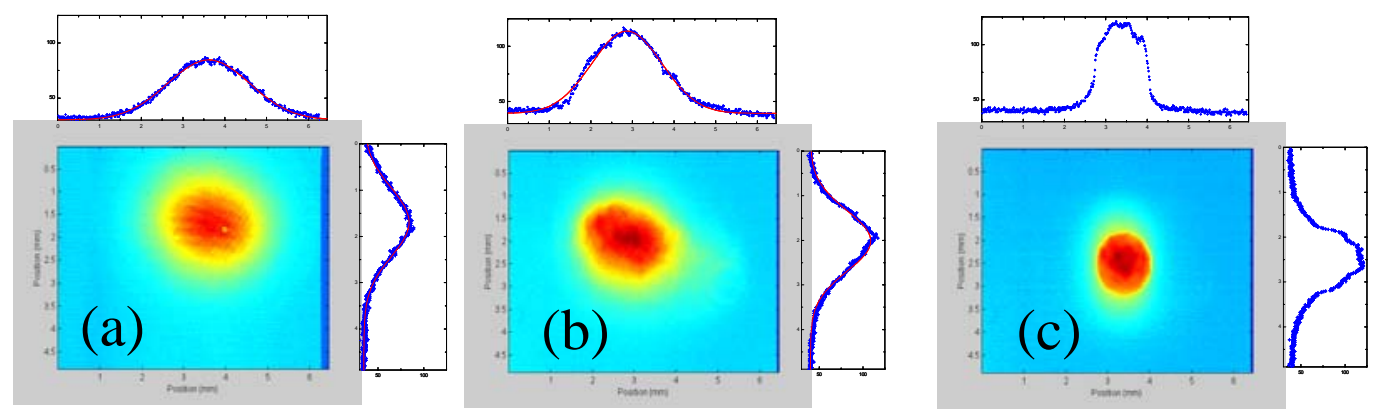

Fig. 6: Beam profile at different stages of transport: (a) after the compressor, $1053 \mathrm{~nm}$; (b) at the virtual cathode, no aperture; (c) at the virtual cathode with a hard edge aperture.

\subsection{Environment control issues}

The laser operation is very sensitive to temperature shift in the environment. Temperature shift on the order of $1-2^{\circ} \mathrm{F}$ normally reduces the beam energy, increases energy instability, and induces significant changes in the beam profile. The impact due to temperature shift on the timing jitter has not yet been characterized.

Currently, the temperature is controlled by forced airflow through the laser room at constant temperature. This way the temperature stability is kept at the $0.5^{\circ} \mathrm{F}$ level.

The vibration of the laser table and transport was measured (Fig. 7) and was found in good shape in comparison with the A0 facility at Fermilab.

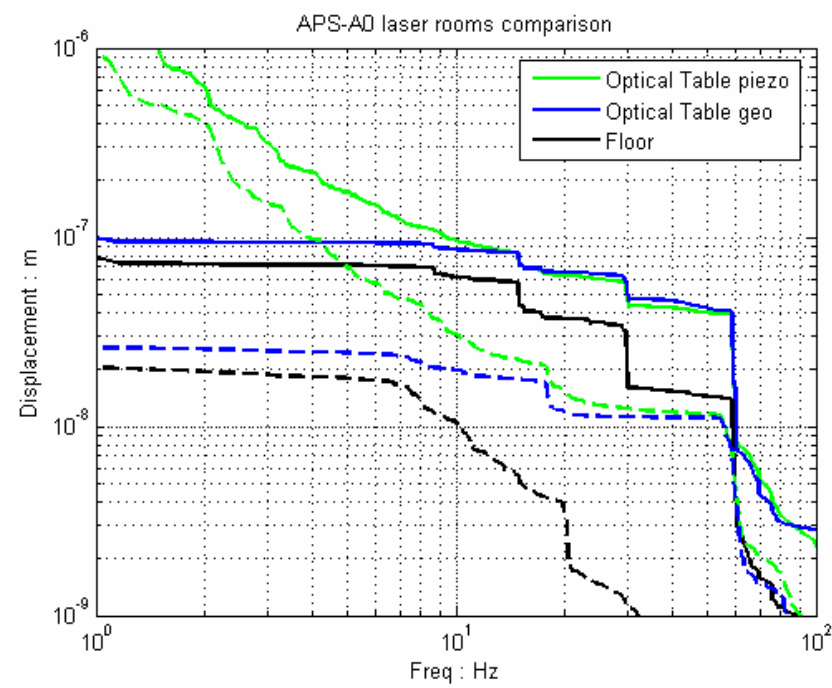

Fig. 7: Environment vibration measurement for the APS photoinjector drive laser and for the Fermi A0 laser environment. Collaboration with J. Li et al. 


\section{Future R\&D and Upgrade Plan}

\subsection{Laser pulse shaping}

Clearly, even though the laser is performing well for our current FEL R\&D, we are still not at the point to meet the requirements of a fourth-generation light source. We still lack fine control of the laser longitudinal and transverse profiles, which has been demonstrated to be critical for significant improvement of the beam quality [8]. We believe this is still a very productive research field to explore.

Though no longitudinal pulse shaping has been planned, collaboration with SLAC is underway to test the transverse profile shaping using a "beam flattener," and its effects on frequency conversion. This is part of the R\&D efforts for the forthcoming LCLS photoinjector drive laser.

\subsection{Adaptive laser control}

A more exciting project is to perform research on an adaptive laser control system, as shown in Fig. 8. In this adaptive system, a computer takes measurements from both the laser and the electron beams and adjusts the laser parameters accordingly to minimize the emittance of the beam. The adaptive control is on both the longitudinal and transverse profiles of the laser beam. The ability to adjust the transverse profile will allow us to eliminate the effect of the QE inhomogeneity from the photocathode.

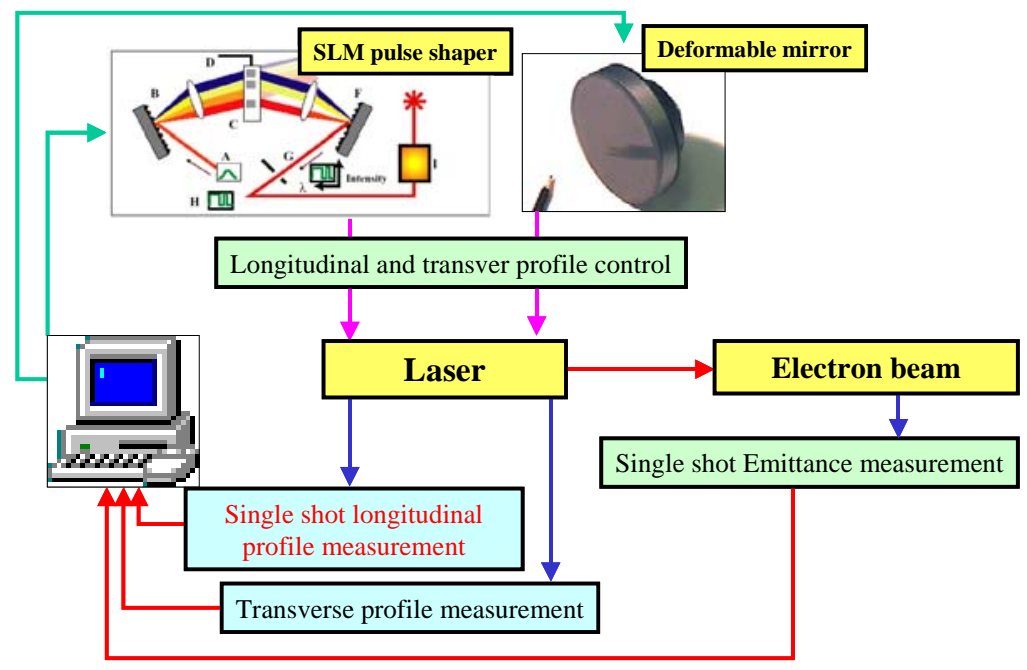

Fig. 8: A schematic of an adaptive controlled laser system for beam optimization from a photoinjector.

\subsection{Other}

Another research opportunity is to push the timing jitter between the laser and rf down to the 10fs level. In contrast, the state-of-the-art jitter is $200 \mathrm{fs}$. 
Electro-optical sampling has been successfully demonstrated to give 600-fs resolution when probing electron bunches; we believe the ongoing research at APS can push the temporal resolution to an even higher level of about $10 \mathrm{fs}$.

\subsection{Upgrade}

To improve the reliability and further improve the stability of the laser system, the laser amplifier needs to be upgraded.

The most plausible upgrade for the laser system is to replace the amplifier heads with diode pumped heads, which will significantly reduce the heat load in the laser cavity, hence reducing the thermal and mechanic stress in the laser rods and reducing or eliminating any possible distortion due to thermal lensing effect. Of course, the maintenance needs will be significantly reduced as well.

\section{Acknowledgements}

Many people have contributed to the work described in this note: Gil Travish (former laser commander), Ned Arnold, Sandra Biedron, Arthur Grelick, Mike Hahne, Kathy Harkay, Rich Kodenhoven, Robert Laird, John Lewellen, Greg Markovich, Stephen Milton, and Anthony Pietryla. This work is supported by the U. S. Department of Energy, Office of Basic Energy Sciences, under Contract No. W-31-109-ENG-38.

\section{References}

1. http://www.slac.stanford.edu/pubs/slacwps/slac-wp-025.html.

2. S. Milton et al., Phys. Rev. Lett. 85, 988 (2000).

3. S. V. Milton et al., Science 292, 2037 (2001).

4. Y. Li et al., Phys. Rev. Lett. 89, 234801 (2002).

5. Y. Li et al., Phys. Rev. Lett. 91, 243602 (2003).

6. V. Sajaev et al., Nucl. Instrum. Methods Phys. Res. A 506, 304 (2003).

7. Y. Li et al., Appl. Phys. B 80, 31 (2004).

8. I. V. Veryovkin et al., Appl. Surf. Sci. 231, 962 (2004).

9. J. Yang et al., J. Appl. Phys. 92, 1608 (2002). 